\title{
Saturation and coherence effects in the modified KGBJS equation
}

\section{Michal Deák*}

Instituto de Física Corpuscular, Universitat de Vàlencia - Consejo Superior de Investigaciones Científicas, Parc Científic, E-46980 Paterna (Valencia), Spain

E-mail: michal.deak@ific.uv.es

\begin{abstract}
We solve the modified non-linear extension of the CCFM equation KGBJS equation numerically for certain initial conditions and compare the resulting gluon Green functions with those obtained from solving the original CCFM equation and the BFKL and BK equations for the same initial conditions. We improve the low transversal momentum behaviour of the KGBJS equation by a small modification.
\end{abstract}

XXI International Workshop on Deep-Inelastic Scattering and Related Subjects 22-26 April, 2013

Marseilles, France

${ }^{*}$ Speaker. 


\section{Introduction}

The BFKL equation seems to be the appropriate effective theory for describing high energy initial state radiation in the kinematic region where the Mandelstam invariants $t$ of the momentum exchange and $s$ of the total scattering momentum are strongly ordered: $\Lambda_{Q C D}^{2} \ll|t| \ll s$. It is well known that the relevant phase space region is very important at the LHC, but signs of its importance were already observed at the HERA and other colliders.

However, the BFKL equation predicts too strong rise of the cross section for decreasing ratio of $|t| / s$ violating the Froisart bound and thus the unitarity. A non-linear extensions of the equation was proposed [1] to take into account merging of over-populated gluons and thus damping the growth of the gluon density and consequently the cross section. The BK equation [2] is one of such extensions of the BFKL equation. The growth of the solution of the BK equation is suppressed compared to the solution of the linear equation.

The BFKL and also the BK equations are only suitable to describe the inclusive cross section. Inclusion of coherence effects was proposed to extend the validity of latter equations for exclusive final states. An equation which includes the BFKL kernel plus the coherence effects and at the same time interpolates between the BFKL and the DGLAP approximations is the CCFM equation [4].

An interesting question raises: How does a corresponding non-linear equation, a non-linear extension of the CCFM equation, look like and how does the coherence requirement interplay with the saturation constraint?

A non-linear extension of the CCFM equation in a simple form was suggested in [3]. The latter proposed non-linear equation was not yet studied in the literature. We will start filling the emerged gap in this publication. We will examine closely the new non-linear equation and find non-physical behaviour near the soft cut-off. We will suggest an improvement of the equation and present numerical results.

\subsection{The CCFM equation}

The CCFM equation reads

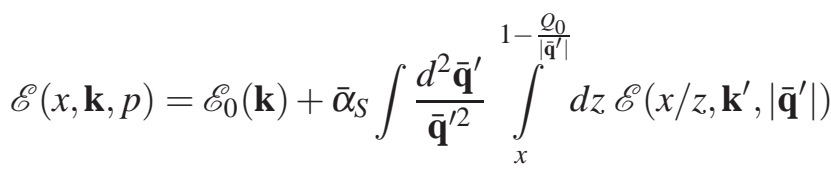

$$
\begin{aligned}
& \times \theta\left(p-z\left|\overline{\mathbf{q}}^{\prime}\right|\right) \mathscr{P}(z, \mathbf{k}, \mathbf{q}) \Delta_{S}\left(p, z\left|\overline{\mathbf{q}}^{\prime}\right|, Q_{0}\right),
\end{aligned}
$$

with $x$ being proton momentum fraction, $\mathbf{k}$ the $t$-channel gluon transversal momentum, $\mathbf{q}$ the transversal momentum of the emitted gluon and $z$ and $\mathscr{E}_{0}(\mathbf{k})$ being now the initial condition. In addition the variable $\overline{\mathbf{q}}=\mathbf{q} /(1-z)=\left(\mathbf{k}^{\prime}-\mathbf{k}\right) /(1-z)$ and a new scale $p$ characterising the hard scale or the maximum emission angle in the evolution are introduced. The function $\mathscr{P}(z, \mathbf{k}, \mathbf{q})$ is the gluon splitting function which includes finite terms.

The factor $\Delta_{S}\left(p,(z \overline{\mathbf{q}})^{2}\right)$ is the Sudakov form factor. The Non-Sudakov form factor $\Delta_{N S}\left(\mathbf{k}^{2},(z \overline{\mathbf{q}})^{2}\right)$ is included in the gluon splitting function. 


\subsection{The KGBJS equation}

We will follow the line of authors of [3] who suggested a non-linear extension of the CCFM equation (1.1) in this form

$$
\begin{aligned}
\tilde{\mathscr{E}}(x, \mathbf{k}, p) & =\widetilde{\mathscr{E}}_{0}(\mathbf{k}) \\
& +\bar{\alpha}_{S} \int \frac{d^{2} \overline{\mathbf{q}}^{\prime}}{\overline{\mathbf{q}}^{\prime 2}} \int_{x}^{1-\frac{Q_{0}}{\overline{\mathbf{q}}^{\prime} \mid}} d z\left(\tilde{\mathscr{E}}\left(x / z, \mathbf{k}^{\prime},\left|\overline{\mathbf{q}}^{\prime}\right|\right)-\delta\left(\overline{\mathbf{q}}^{\prime 2}-\mathbf{k}^{2}\right)\left(\overline{\mathbf{q}}^{2}\right) \tilde{\mathscr{E}}^{2}\left(x / z, \overline{\mathbf{q}}^{\prime},\left|\overline{\mathbf{q}}^{\prime}\right|\right)\right) \\
& \times \theta\left(p-z\left|\overline{\mathbf{q}}^{\prime}\right|\right) \mathscr{P}(z, \mathbf{k}, \mathbf{q}) \Delta_{S}\left(p, z\left|\overline{\mathbf{q}}^{\prime}\right|, Q_{0}\right) .
\end{aligned}
$$

An important comment is required about the upper limit in the $z$ integral in (1.1). This upper limit regulates integrals with a pole in $z$. It is easy to see, that it also generates a limit on the value of the variable $\left|\mathbf{q}^{\prime}\right|>Q_{0} /(1-z)$ and also on the variable $|\overline{\mathbf{q}}|>Q_{0} /(1-z)$. The latter limit is going to affect transversal momentum dependence of the solution of the KGBJS equation (1.2) near the soft scale $|\mathbf{k}| \sim Q_{0}$. In case when $Q_{0}<|\mathbf{k}|<Q_{0} /(1-z)$ the non-linear term in the (1.2) will be equal to 0 rendering the solutions of the linear and the non-linear equations almost equal. For $|\mathbf{k}|=Q_{0}$ we thus have

$$
\tilde{\mathscr{E}}\left(x, Q_{0}, p\right) \simeq \mathscr{E}\left(x, Q_{0}, p\right) .
$$

This is not what we would intuitively expect from a dipole amplitude with growth tamed by a non-linear correction. We are going to study properties of the solutions of the non-linear equation (1.2), but we suggest a modification which can give a more natural behaviour of its solution. We modify the argument of the delta function in the non-linear term

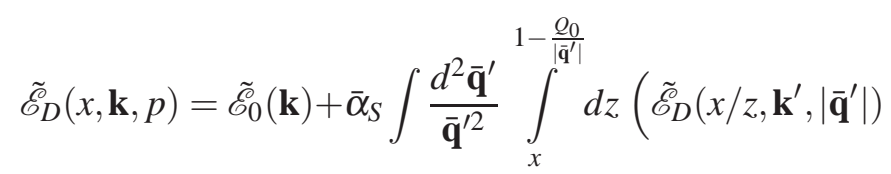

$$
\begin{aligned}
& \left.-\delta\left(\overline{\mathbf{q}}^{\prime 2}-\mathbf{k}^{2} /(1-z)^{2}\right)\left(\overline{\mathbf{q}}^{\prime 2}\right) \tilde{\mathscr{E}}_{D}^{2}\left(x / z, \overline{\mathbf{q}}^{\prime},\left|\overline{\mathbf{q}}^{\prime}\right|\right)\right) \\
& \times \boldsymbol{\theta}\left(p-z\left|\overline{\mathbf{q}}^{\prime}\right|\right) \mathscr{P}(z, \mathbf{k}, \mathbf{q}) \Delta_{S}\left(p, z\left|\overline{\mathbf{q}}^{\prime}\right|, Q_{0}\right)
\end{aligned}
$$

to shift its 'pole' outside of the interval $\left(Q_{0}, Q_{0} /(1-z)\right)$.

\section{Numerical solutions of the equations: Discussion of the results}

We solve the equations (1.1), (1.2) and (1.4) by iteration on a lattice under certain additional conditions. We set the $\bar{\alpha}_{S}=0.2$. We have written the CCFM equation with a form of the NonSudakov form factor which requires a kinematical constraint to satisfy unitarity. We will thus require $|\mathbf{q}|<|\mathbf{k}| / \sqrt{z}$ [5] in the CCFM kernel. We set the parameters $\mu=Q_{0}=1 \mathrm{GeV}$. To mimic energy-momentum conservation we apply an upper limit on the $\overline{\mathbf{q}}, \mathbf{q}<\sqrt{s_{\text {tot }}}$ integration. In this publication we choose $\sqrt{s_{t o t}} \simeq\left(1 \mathrm{GeV} / x_{\min }\right)$. Where $x_{\min }$ is the minimal momentum fraction $x$ for which we parametrise the solution of a given equation. We take the initial condition to be

$$
\tilde{\mathscr{E}}^{0}\left(\mathbf{k}^{2}\right)=\frac{C_{\text {in }}}{|\mathbf{k}|}
$$

with $C_{i n}$ being a constant parameter. We set $C_{i n}=0.5$ for the CCFM and the KGBJS equations. 


\subsection{Discussion}

In this subsection we are going to discuss the comparison of the solution of the CCFM equation (1.1) with our new modified KGBJS equation (1.4).

Let us take a look on the plots in the figure 2. We will first discuss the $\mathbf{k}$ distributions. We can see that the modification really breaks the condition (1.3), so the solutions of the linear equation and the non-linear equation are not equal at the soft cut-off $Q_{0}$. We do not observe a formation of the dip as for the original KGBJS equation in figure 1. The behaviour of the suppression for small $\mathbf{k}$ is similar to the one generated by the BK equation.

The natural behaviour of the solution of (1.4) is reflected also in the $x$ distributions. The difference between $x$ distribution of the CCFM and the non-linear equation gets smaller for $|\mathbf{k}|$ getting bigger.

\section{Conclusions}

We have numerically obtained solutions of different versions of the CCFM equation. We have also solved the KGBJS equation [3] and its modification.

We have studied the transversal momentum $\mathbf{k}$ and $x$ distributions of the obtained solutions analytically and also numerically.

We find that solutions of the CCFM and the KGBJS equations match at the soft cut-off which implies no suppression in the point where the CCFM solution has the biggest magnitude.

We suggest a modification of the KGBJS equation which removes the unintuitive behaviour of the solutions of the original equation near the soft cut-off.

The resulting suppression due to the non-linear term in the solution of equations (1.2) and (1.4) is a result of complicated interplay between values of the dipole amplitude in the small $x$ and also large $x$ phase space regions.

Although the investigation presented here shows, that it is not easy to find a natural model for a non-linear extension of the CCFM equation, we recommend the improved equation (1.4) to be a subject of more studies of inclusive and exclusive observables.

\section{Acknowledgements}

MD acknowledges support from Juan de la Cierva programme.

\section{References}

[1] A. H. Mueller, Parton distributions at very small x values, Nucl. Phys. Proc. Suppl. 18C (1991) 125-132.

[2] Y. V. Kovchegov, Small x F(2) structure function of a nucleus including multiple pomeron exchanges, Phys.Rev. D60 (1999) 034008, [hep-ph/9901281].

Y. V. Kovchegov, Unitarization of the BFKL pomeron on a nucleus, Phys.Rev. D61 (2000) 074018, [hep-ph/9905214].

I. Balitsky, Operator expansion for high-energy scattering, Nucl.Phys. B463 (1996) 99-160, [hep-ph/9509348]. 
[3] K. Kutak, Nonlinear extension of the CCFM equation, arXiv: 1206.1223.

K. Kutak, K. Golec-Biernat, S. Jadach, and M. Skrzypek, Nonlinear equation for coherent gluon emission, JHEP 1202 (2012) 117, [arXiv:1111.6928].

[4] M. Ciafaloni, Coherence effects in initial jets at small $q^{2} / s$, Nucl. Phys. B296 (1988) 49.

S. Catani, F. Fiorani, and G. Marchesini, QCD coherence in initial state radiation, Phys. Lett. $\mathbf{B 2 3 4}$ (1990) 339.

S. Catani, F. Fiorani, and G. Marchesini, Small x behavior of initial state radiation in perturbative QCD, Nucl. Phys. B336 (1990) 18.

G. Marchesini, QCD coherence in the structure function and associated distributions at small x, Nucl. Phys. B445 (1995) 49.

[5] E. Avsar and E. Iancu, CCFM Evolution with Unitarity Corrections, Nucl. Phys. A829 (2009) 31-75, [arXiv:0906.2683].

E. Avsar and A. M. Stasto, Non-linear evolution in CCFM: The Interplay between coherence and saturation, JHEP 1006 (2010) 112, [arXiv: 1005 . 5153].
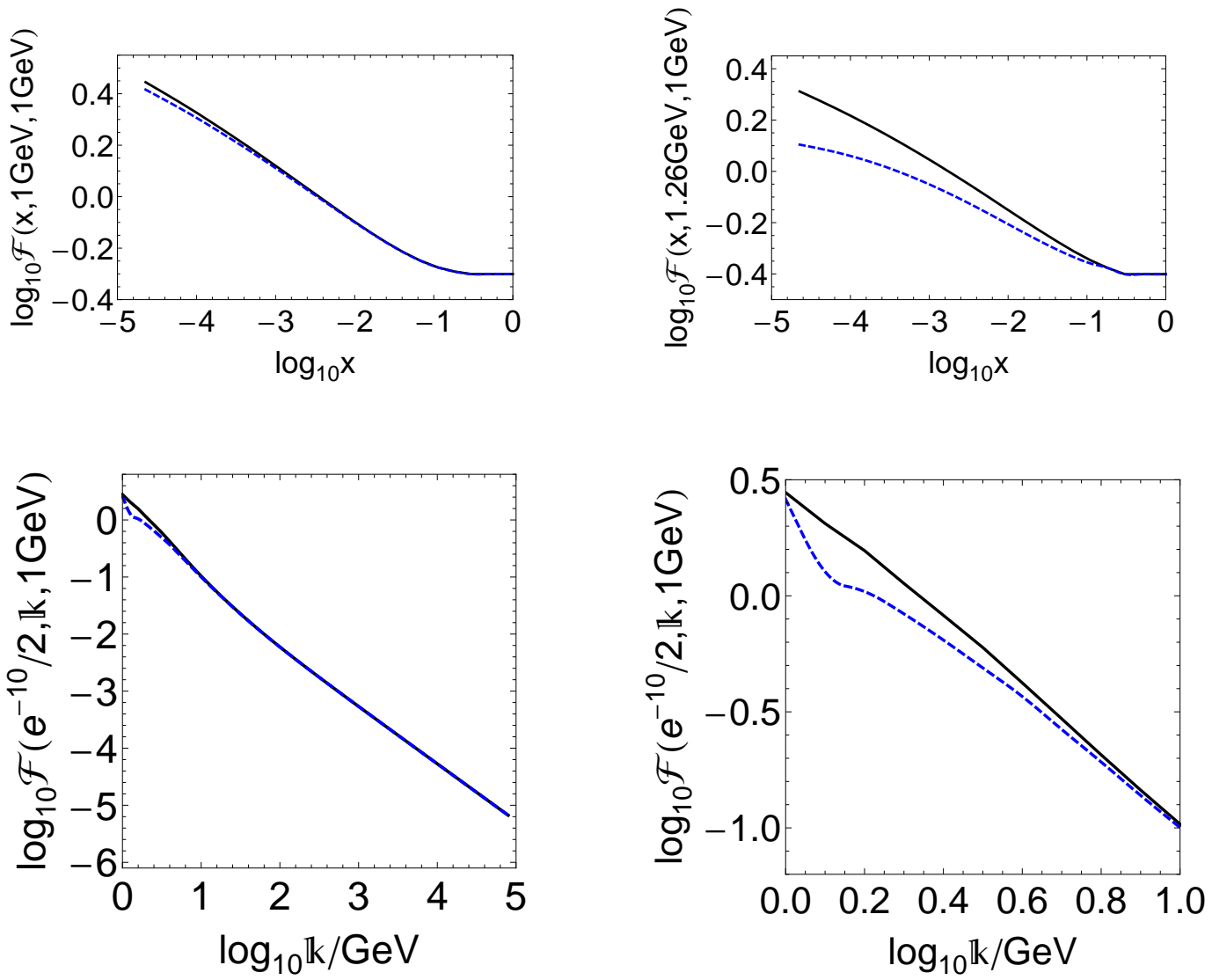

Figure 1: The $x$ and $\mathbf{k}$ distributions of different versions of the CCFM equation - the solid black line compared with the KGBJS equation (1.2) - the blue dashed line. 

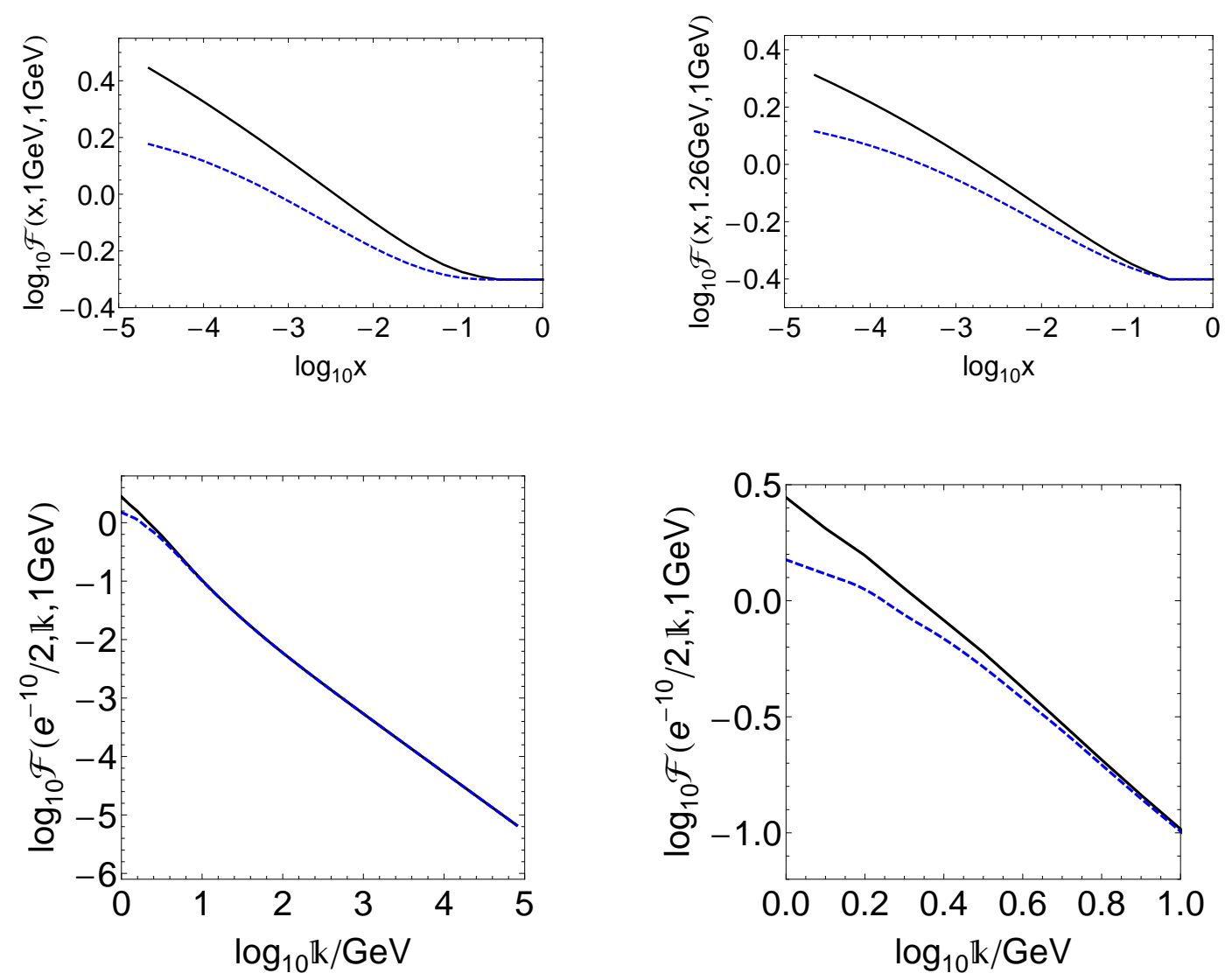

Figure 2: The $x$ and $\mathbf{k}$ distributions of different versions of the CCFM equation - the solid black line compared with the modified KGBJS equation (1.4) - the blue dashed line. 